\title{
Drude Weight of the Two-Dimensional Hubbard Model - Reexamination of Finite-Size Effect in Exact Diagonalization Study -
}

\author{
Hiroki Nakano*, Yoshinori Takahashi and Masatoshi Imada ${ }^{1}$ \\ Graduate School of Material Science, University of Hyogo, Kouto 3-2-1, Kamiori, Ako-gun, 678-1297 \\ ${ }^{1}$ Department of Applied Physics, University of Tokyo, Hongo 7-3-1, Tokyo 113-8656
}

(Received October 2, 2018)

\begin{abstract}
The Drude weight of the Hubbard model on the two-dimensional square lattice is studied by the exact diagonalizations applied to clusters up to 20 sites. We carefully examine finite-size effects by consideration of the appropriate shapes of clusters and the appropriate boundary condition beyond the limitation of employing only the simple periodic boundary condition. We successfully capture the behavior of the Drude weight that is proportional to the squared hole doping concentration. Our present result gives a consistent understanding of the transition between the Mott insulator and doped metals. We also find, in the frequency dependence of the optical conductivity, that the mid-gap incoherent part emerges more quickly than the coherent part and rather insensitive to the doping concentration in accordance with the scaling of the Drude weight.
\end{abstract}

KEYWORDS: Hubbard model, Lanczos method, metal-insulator transition, Drude weight, optical conductivity

\section{Introduction}

Metal-insulator transition is a fundamental issue in the condensed-matter physics. Particular interest has been focused on properties of metals near the transition between the Mott insulator and the metallic phase. Many studies have already been made from both theoretical and experimental points of view. ${ }^{1}$

One of the quantities by which one can distinguish between metallic or insulating phases is the Drude weight $D$, namely the coherent component of the optical conductivity $\sigma(\omega)$. The system is metallic or insulating depending on $D \neq 0$ or $D=0$, respectively. In the Mott insulator, the Drude weight therefore always vanishes. If one dopes the Mott insulator with carriers, it becomes metallic and the Drude weight increases with further doping.

The Hubbard model is by far the simplest microscopic model which can show the Mott-insulator-metal transition. It is the most appropriate model for intensive theoretical studies. Precise determination of its properties by some methods with no approximations contributes much to the fundamental understanding of the metalinsulator transition. In spite of the simplicity, various aspects of typical many-body problems are involved in this model due to the presence of the Coulomb interaction. Many of them are still controversial even now. Among them is the hole-doping dependence of the Drude weight near the transition between the Mott insulator and the metal. Primary interest of this paper is the dependence of the two-dimensional Hubbard model for small doping concentration; this is because the Mott insulator of this model at half filling is considered to become metallic in a nontrivial way when the doping is switched on and because this model has been extensively studied as a model of high- $T_{\mathrm{c}}$ cuprates.

Generally, it is not so easy to calculate such a dynam-

*E-mail address: hnakano@sci.u-hyogo.ac.jp ical quantity as the Drude weight of the strongly correlated system without approximations. One possible way is to apply the exact diagonalization method to finitesize clusters. The first work along this line concerning with the two-dimensional Hubbard model was reported by Dagotto et $a .^{2}$ Based on the treatment on the clusters up to 16 sites, they successfully captured a rough feature of the Drude weight as a function of the hole doping concentration $\delta$. They tentatively concluded that $D \propto \delta$, although the Drude weight sometimes becomes negative with large absolute values especially near the metalinsulator transition point. Such negative Drude weights occur as a consequence of the finite-size effect. In order to get rid of such a finite-size effect, Nakano and Imada examined the properties under various boundary conditions in the system of the same size with 16 sites. ${ }^{3}$ They consequently found the concave behavior of the Drude weight as a function of $\delta$ in the small- $\delta$ region, which implies the dependence $D \propto \delta^{2}$. Recently, Tohyama and his co-workers treated larger clusters of 18 and 20 sites under the periodic boundary condition. ${ }^{4}$ Their result of the Drude weight seems to suggest $D \propto \delta$ again. Thus, the issue of the $\delta$-dependence of $D$ is still controversial at present.

Under these circumstances, we examine the finite-size effects of the Drude weight of clusters with various shapes and sizes up to 20 sites under various boundary conditions very carefully and do our best in minimizing the finite-size effects when we calculate the Drude weight of the finite-size clusters. The purpose of the study in this paper is to capture thereby a reliable exponent for the $\delta$-dependence of the Drude weight near the transition between the metal and the Mott insulator. Our present results suggest that $D \propto \delta^{2}$.

This paper is organized as follows. In the next section, the model Hamiltonian and the method of numerical calculation are introduced. Section 3 is devoted to the ex- 
amination of the finite-size effect in the non-interacting case. In Section 4, results in the interacting case are reported and discussed. Summary and conclusion are given in the final section.

\section{Model and Method}

We consider the two-dimensional Hubbard model. Its Hamiltonian is given by

$$
\begin{aligned}
\mathcal{H} & =\mathcal{H}_{\text {hop }}+\mathcal{H}_{\text {int }}, \\
\mathcal{H}_{\text {hop }} & =-t \sum_{\langle i, j\rangle, \sigma} c_{i, \sigma}^{\dagger} c_{j, \sigma}, \\
\mathcal{H}_{\text {int }} & =U \sum_{i} n_{i \uparrow} n_{i \downarrow},
\end{aligned}
$$

where the creation (annihilation) of an electron at site $i$ with spin $\sigma$ is denoted by $c_{i, \sigma}^{\dagger}\left(c_{i, \sigma}\right)$ and $n_{i, \sigma}$ is the corresponding number operator, namely $n_{i, \sigma}=c_{i, \sigma}^{\dagger} c_{i, \sigma}$. In this paper, we treat only the nearest-neighbor hopping on the two-dimensional square lattice. Shapes for finite-size clusters will be illustrated later. Energies are measured in units of $t$; therefore, we set $t=1$ hereafter. The system consists of $N_{\mathrm{e}}$ electrons on $N_{\mathrm{s}}$ atomic sites. The hole doping concentration is a controllable parameter given by $\delta=1-n$, where the electron density is defined as $n=N_{\mathrm{e}} / N_{\mathrm{s}}$.

We perform numerical diagonalization of finite-size clusters of the model (1) using Lanczos algorithm. This method is non-biased against the effects of Coulomb interaction. The method is also useful to obtain the ground-state wave function with the ground-state energy $E_{\mathrm{g}}$ very precisely. On the other hand, the disadvantage of this method is that available system sizes are limited to be small. This is because the dimension of the Hilbert space grows exponentially with respect to the system size. ${ }^{5}$ In order to overcome this disadvantage, we perform parallel calculations in the computer systems of the distributed-memory type. Thereby we can treat arbitrary shapes of clusters up to $N_{\mathrm{s}}=20$ if we use appropriate supercomputer systems. We also employ the continued-fraction expansion method, ${ }^{6}$ which provides us with dynamical quantities including the optical conductivity and the Drude weight. The detailed procedure will be explained after the examinations of cluster shapes and boundary conditions.

\section{Finite-Size Effect for $U=0$ and Boundary Condition}

In this section, we examine how finite-size effects occur in various types of boundary conditions of clusters for a given $N_{\mathrm{s}}$. When one considers the system of twodimensional square lattice by using a finite-size cluster, regular squares under the periodic boundary condition are usually adopted as in refs. 2 and 4 . Such regular squares for $N_{\mathrm{s}}=16,18$ and 20 are depicted in Fig. 1 (a), (b) and (c), respectively. The merit of adopting these squares is that the $x$ and $y$ directions are equivalent. When one treats such a quantity as the Drude weight, however, calculations based on the regular squares under the periodic boundary condition reveal a large finite-size

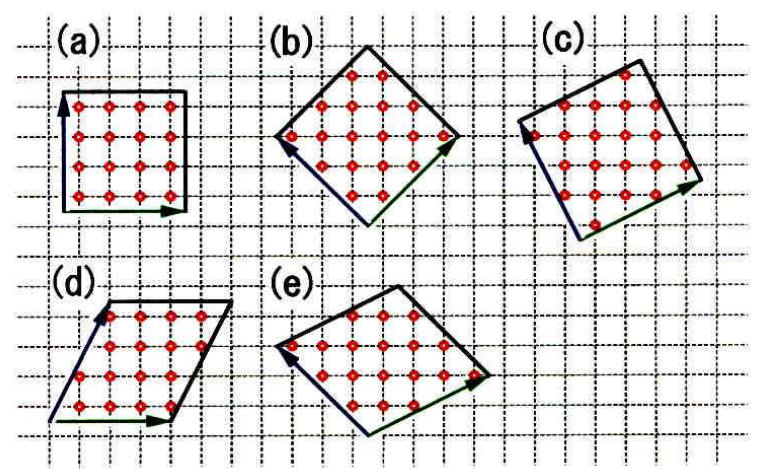

Fig. 1. Finite size clusters for $N_{\mathrm{S}}=16,18$ and 20. The blue and green vectors represent two translational vectors for each cluster. The atomic sites are denoted by the red circles inside the quadrangle formed by these two vectors and the other edges denoted by black solid lines.

deviation from the value in the thermodynamic limit. In ref. 2, a remarkable finite-size effect was reported. In view of this situation, effects of other types of boundary conditions, namely the antiperiodic boundary condition and the mixed boundary condition, were studied in ref. 3 to see whether one can reduce the finite-size effects for the fixed $N_{\mathrm{s}}$ or not. Consequently, the wrong negative large Drude weights were successfully resolved. Note here that when one imposes the mixed boundary condition, the two lattice directions are no longer equivalent and the average of the two directions gives a reasonable result. This suggests that adhering to the equivalence of the two directions is not necessary. The two directions become inequivalent when one relaxes the condition that these quadrangles of the clusters have the right angle at their vertices. Therefore we rather additionally examine the parallelogram clusters depicted in Fig. 1 (d) and (e) for $N_{\mathrm{s}}=16$ and 18 in place of squared-shaped clusters, respectively. An important point is that a set of possible wave number vectors corresponding to the clusters (d) and (e) is different from the one corresponding to the clusters (a) and (b), respectively. The system is nevertheless still bipartite. For $N_{\mathrm{s}}=20$, on the other hand, it is impossible to preserve the bipartite nature when one distorts the cluster (c) so that it realizes a parallelogram cluster with a different set of possible wave number vectors. Then we study only one type of cluster for $N_{\mathrm{s}}=20$. Therefore we consider these clusters (a)-(e) up to $N_{\mathrm{s}}=20$.

Next, we show how to select the appropriate cluster under the appropriate boundary condition for a given $N_{\mathrm{s}}$. For this purpose, let us first see the ground-state energy $E_{\mathrm{g}}$ of the clusters (a)-(e) at half filling for $U=0$, presented in Table I. Note that this quantity is proportional to the Drude weight for $U=0$. A noticeable point of the results of clusters (a), (b) and (c) under the periodic boundary condition is that the relative difference $\left(E_{\infty}-E_{\mathrm{g}}\right) / E_{\infty}$ does not monotonically converge as $N_{\mathrm{s}}$ is increased, where $E_{\infty}$ denotes the ground-state energy in the limit of $N_{\mathrm{s}} \rightarrow \infty$. This suggests that the system size $N_{\mathrm{s}}$ up to 20 is not free from the finite-size effect as 
far as we stick to the periodic boundary condition. We have thus to be careful in choosing an appropriate cluster under an appropriate boundary condition for a given $N_{\mathrm{s}}$. Let us now compare the difference $\left(E_{\infty}-E_{\mathrm{g}}\right) / E_{\infty}$ of all the clusters (a)-(e) with various boundary conditions. One can find the best cluster and boundary condition from among the cases shown in Table I so that the absolute value of the difference is the smallest. For $N_{\mathrm{s}}=16$ and 20, two boundary conditions give the smallest one. Sets of possible wave number vectors of the two cases for each $N_{\mathrm{s}}$ are equivalent. Thus, the same result will be obtained if one selects either of the two.

Let us compare the $\delta$-dependence of the noninteracting $E_{\mathrm{g}}$ between two cases, the one of the most appropriate cluster under the most appropriate boundary condition with the best $E_{\mathrm{g}}$ at half filling and the other of the regular square under the periodic boundary condition; results are depicted in Fig. 2. One can easily observe the results of the former case fall closer to the curve of the thermodynamic limit in the whole region of $\delta$, irrespective of the size $N_{\mathrm{s}}$. On the other hand, the latter regular-square case under the periodic boundary condition gives significant overestimates particularly at $\left(N_{\mathrm{s}}\right.$, $\left.N_{\mathrm{e}}\right)=(18,18)$ and $(20,18)$. This strongly suggests that the Drude weights of these cases will be possibly overestimated even when $U$ is considerably large. It also suggests that if the intrinsic Drude weight shows the concave behavior as a function of $\delta$ for large $U$, this overestimation will possibly cancel this behavior. Figure 2 also provides another important finite-size effect that finite-size data

Table I. List of ground-state energy per site in the noninteracting case $U=0$ at half filling for various shapes of clusters and various boundary conditions together with relative difference from the energy in the thermodynamic limit. Head $\mathrm{BC} 1$ (BC2) denotes the boundary condition along the green (blue) translational vector in Fig. 1. P and A represent the periodic boundary condition and the antiperiodic boundary condition, respectively. Note here that the boundary conditions giving the same energy for each cluster correspond to the identical set of possible wave number vectors. Marked cases in last column with head PW show the selected clusters under the selected boundary condition in which we actually perform calculations in the present work.

\begin{tabular}{c|cc|lc|c}
\hline cluster & $\mathrm{BC} 1$ & $\mathrm{BC} 2$ & $-E_{\mathrm{g}} / N_{\mathrm{S}}$ & $\left(E_{\infty}-E_{\mathrm{g}}\right) / E_{\infty}$ & $\mathrm{PW}$ \\
\hline (a) & $\mathrm{P}$ & $\mathrm{P}$ & 1.5 & -0.0747 & \\
& $\mathrm{~A}$ & $\mathrm{P}$ & 1.70711 & +0.0530 & \\
& $\mathrm{P}$ & $\mathrm{A}$ & 1.70711 & +0.0530 & \\
& $\mathrm{~A}$ & $\mathrm{~A}$ & 1.41421 & -0.1276 & \\
\hline (b) & $\mathrm{P}$ & $\mathrm{P}$ & 1.77778 & +0.0966 & \\
& $\mathrm{~A}$ & $\mathrm{P}$ & 1.53960 & -0.0503 & \\
& $\mathrm{P}$ & $\mathrm{A}$ & 1.53960 & -0.0503 & \\
& $\mathrm{~A}$ & $\mathrm{~A}$ & 1.33333 & -0.1775 & \\
\hline (c) & $\mathrm{P}$ & $\mathrm{P}$ & 1.69443 & +0.0452 & \\
& $\mathrm{~A}$ & $\mathrm{P}$ & 1.65065 & +0.0182 & $\bullet$ \\
& $\mathrm{P}$ & $\mathrm{A}$ & 1.65065 & +0.0182 & \\
& $\mathrm{~A}$ & $\mathrm{~A}$ & 1.52169 & -0.0613 & \\
\hline (d) & $\mathrm{P}$ & $\mathrm{P}$ & 1.70711 & +0.0530 & \\
& $\mathrm{~A}$ & $\mathrm{P}$ & 1.63099 & +0.0061 & $\bullet$ \\
& $\mathrm{P}$ & $\mathrm{A}$ & 1.5 & -0.0747 & \\
& $\mathrm{~A}$ & $\mathrm{~A}$ & 1.63099 & +0.0061 & \\
\hline (e) & $\mathrm{P}$ & $\mathrm{P}$ & 1.72417 & +0.0636 & \\
& $\mathrm{~A}$ & $\mathrm{P}$ & 1.64519 & +0.0148 & $\bullet$ \\
& $\mathrm{P}$ & $\mathrm{A}$ & 1.51621 & -0.0647 & \\
& $\mathrm{~A}$ & $\mathrm{~A}$ & 1.44675 & -0.1076 & \\
\hline
\end{tabular}
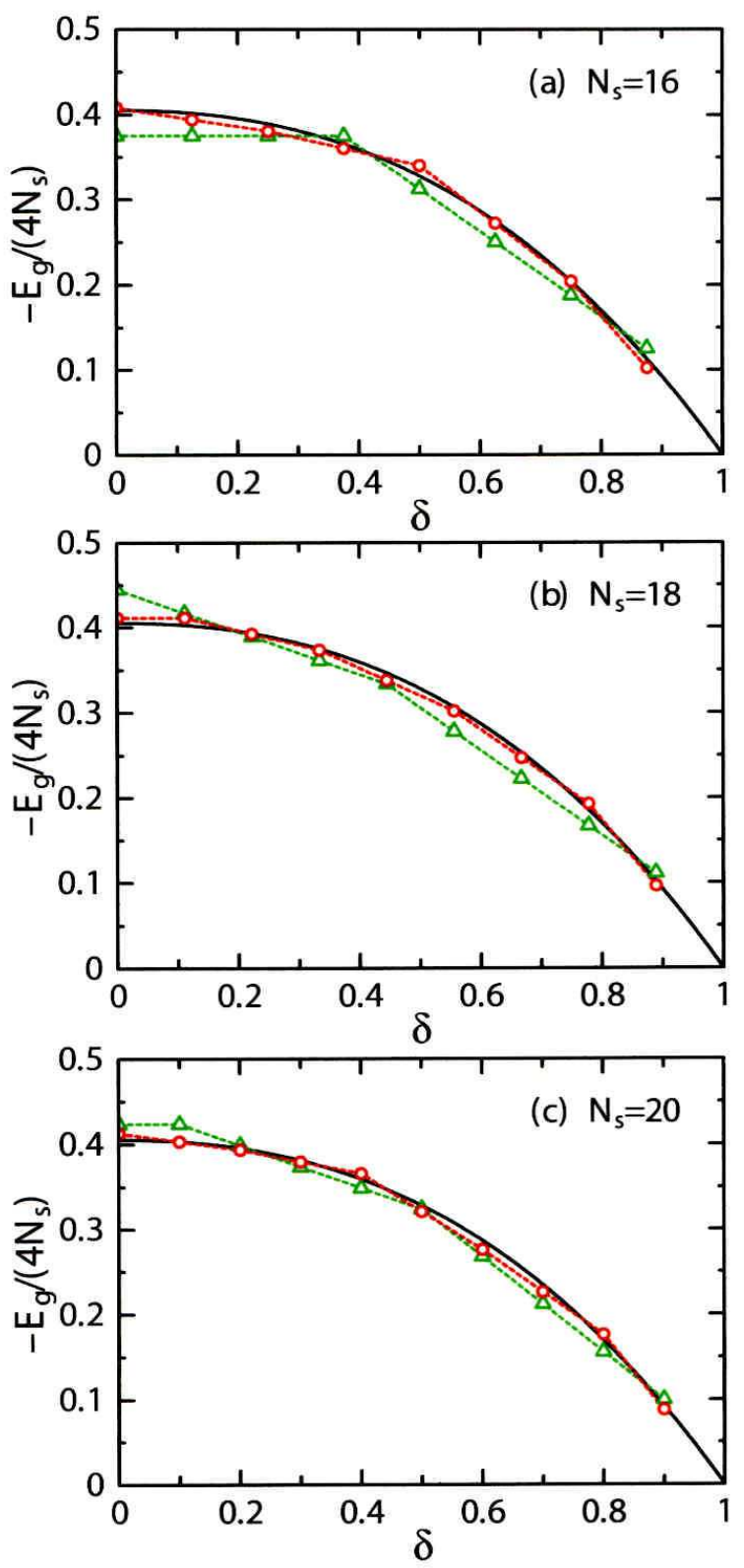

Fig. 2. Ground-state energies as function of $\delta$ in non-interacting case for given $N_{\mathrm{s}}$ presented in the form of $-E_{\mathrm{g}} /\left(4 N_{\mathrm{s}}\right)$. This quantity corresponds to $D$ for $U=0$. Green triangles represent data for the regular square under the periodic boundary condition. Red circles represent data for the appropriate cluster under the appropriate boundary condition based on the analysis in Table I. The solid curve denotes the corresponding quantity for the thermodynamic limit.

show nonzero curvature in their $\delta$-dependence only at $\delta$ corresponding to the closed electronic shell structure. At $\delta$ for the open electronic shell structure, on the other hand, $E_{\mathrm{g}}$ follows linear dependence. The linear dependence from this origin is likely to survive more or less even for $U>0$. This is, in fact, confirmed in the quantum Monte Carlo study at nonzero $U .{ }^{7}$ Thus, it is difficult to judge whether the linear $\delta$-dependence revealed from numerical studies in the interacting case in the region of the open shell structure is intrinsic or is simply attributed to this particular electronic structure. In view of this situation, it is desirable to confine our analysis to the data 
at $\delta$ for the closed shell structure in order to extract the intrinsic behavior. In this paper, therefore, we take the appropriate clusters under the the appropriate boundary condition for each $N_{\mathrm{s}}$ (shown in Table I) and adopt data for the filling forming the closed shell structure.

In the selected clusters and boundary conditions, the equivalence of the two directions denoted by $\alpha(=x, y)$ does not hold, as we have already mentioned in the above. Then, we take the direction average according to the procedure used in ref. 3. The optical conductivity is given by $\sigma(\omega)=2 \pi D \delta(\omega)+\sigma^{\mathrm{reg}}(\omega)$. Here, the second term is the regular part of the optical conductivity, which is also called the incoherent part. The regular part $\sigma^{\mathrm{reg}}(\omega)$ is obtained by the average $\sigma^{\mathrm{reg}}(\omega)=\left[\sigma_{x}^{\mathrm{reg}}(\omega)+\sigma_{y}^{\mathrm{reg}}(\omega)\right] / 2$; each of $\sigma_{\alpha}^{\text {reg }}(\omega)$ given by

$$
\sigma_{\alpha}^{\mathrm{reg}}(\omega)=\frac{\pi}{N_{\mathrm{s}}} \sum_{\ell(\neq 0)} \frac{\left|\left\langle\ell\left|j_{\alpha}\right| 0\right\rangle\right|^{2}}{E_{\ell}-E_{0}} \delta\left(\omega-E_{\ell}+E_{0}\right),
$$

is calculated by the continued-fraction expansion method. Here, $j_{\alpha}$ is a current operator along the $\alpha$ direction defined as

$$
j_{\alpha}=-\mathrm{i} \sum_{i, \sigma} t\left(c_{i, \sigma}^{\dagger} c_{i+\delta_{\alpha}, \sigma}-c_{i+\delta_{\alpha}, \sigma}^{\dagger} c_{i, \sigma}\right),
$$

where $\delta_{\alpha}$ is the unit vector along the $\alpha$-direction, $|\ell\rangle$ represents an eigenstate with the energy eigenvalue $E_{\ell}$. Note that the ground state is described by $\ell=0$. One can obtain the Drude weight $D$ from the combination of $\sigma(\omega)$ and the sum rule

$$
\int_{0}^{\infty} \sigma(\omega) \mathrm{d} \omega=\pi K
$$

where $-4 K$ represents the kinetic energy per site, namely $-4 K=\left\langle\mathcal{H}_{\text {hop }}\right\rangle / N_{\mathrm{s}}$. For $U=0$, the incoherent part is absent; therefore one has $D=K$. It is widely known that for $U>0$, the incoherent part appears. When $U$ is large enough, one can easily recognize in $\sigma(\omega)$ the responses toward the upper-Hubbard band. In such a case, one can definitely determine a frequency just below the upperHubbard band to be $\omega_{\mathrm{c}}$; we also calculate definite integral

$$
N_{\text {eff }}=\frac{1}{\pi} \int_{0}^{\omega_{\mathrm{c}}} \sigma(\omega) \mathrm{d} \omega,
$$

which is called an effective carrier density.

As for the validity of extending the present procedure to cases with finite $U$, there is no convincing theoretical argument on the condition of reducing the finite-size effect. Though not reliable in a strict and rigorous sense, it is at least better to start from situations where the effects are reduced in the case of $U=0$ from the continuity of behavior as the function of $U$. Another evidence for the validity of the present procedure is given from our examination of $E_{\mathrm{g}}$ for $U>0$; the results are depicted in Fig. 3 . Although all the possible data of $N_{\mathrm{s}}=16,18$ and 20 are shown there with the same closed symbol for each case of $U>0, \delta$-dependence of $E_{\mathrm{g}}$ is fairly smooth. This fact implies that finite-size deviations of the present data are small. One can actually confirm that the present results for $U=4$ agree well with the results from the quantum Monte Carlo simulations ${ }^{7}$ on larger systems up to $N_{\mathrm{s}}=12 \times 12$. Therefore, it is reasonable to presume that

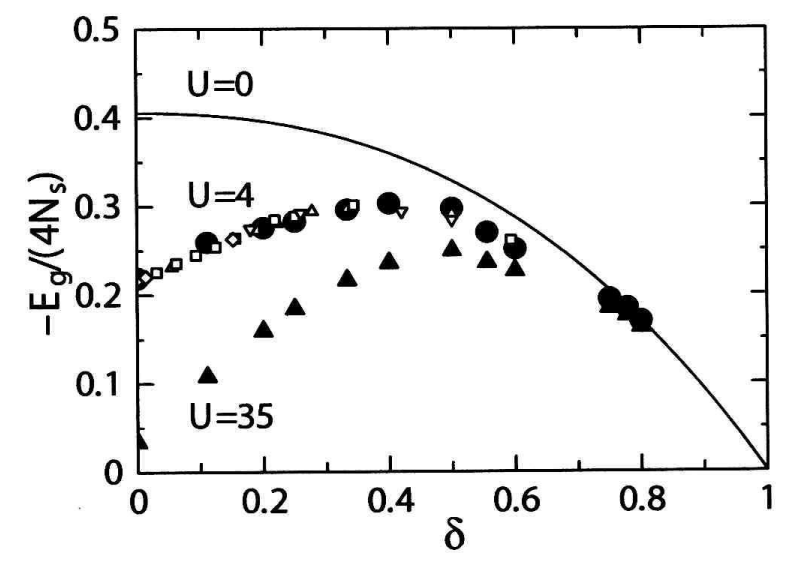

Fig. 3. Ground-state energies as function of $\delta$ in interacting cases presented in the form of $-E_{\mathrm{g}} /\left(4 N_{\mathrm{s}}\right)$. The solid curve denotes $-E_{\mathrm{g}} /\left(4 N_{\mathrm{s}}\right)$ for $U=0$ for the thermodynamic limit. Closed circles and closed triangles denote the present results from exact diagonalization calculations for $U=4$ and $U=35$, respectively. Open symbols (triangles, squares, reversed triangles and diamonds) denote results of $N_{\mathrm{s}}=6 \times 6,8 \times 8,10 \times 10$ and $12 \times 12$ for $U=4$ from the quantum Monte Carlo simulations, respectively. ${ }^{7}$

the finite-size effects in $D$ and $N_{\text {eff }}$ based on the present procedure are also small if the smooth $\delta$-dependence of $D$ and $N_{\text {eff }}$ is confirmed irrespective of the size $N_{\mathrm{s}}$.

In this paper, we take three cases of $U=10,20$ and 35 . In the system with a nonzero $U$ weaker than $U=10$, it is difficult to distinguish the upper-Hubbard band and the lower-Hubbard band in the responses of $\sigma(\omega)$; the estimation of $N_{\text {eff }}$ is not available. On the other hand, in the system for $U>35$, the ground state is possibly ferromagnetic near half filling. Once the ferromagnetism appears, it is too difficult to study the scaling of $D$ near half filling.

\section{Results for Interacting Case and Discussions}

We show numerical results for the $\delta$-dependence of $N_{\text {eff }}$ connected by lines for interacting cases in Fig. 4. The $\delta$ dependence of the Drude weight is later shown. Although the data consist of those of various system sizes for each case of $U, \delta$-dependence of them is fairly smooth, independent of the value $U$, as shown in Fig. 4(a). It clearly indicates that deviations of our numerical results due to the finite-size effect are very small for every $U$ that we treat. One can also observe that as $\delta$ is decreased toward the half-filling case $\delta=0, N_{\text {eff }}$ goes to a very small value for each of non-zero $U$. The values of $N_{\text {eff }}$ at half filling are very close to the corresponding $D$. This is because no significant responses within $0<\omega<\omega_{\text {c }}$ are observed in the optical conductivity; this behavior has already been reported in all the previous works ${ }^{2-4}$ and will be confirmed later in the present study. Then, we will later come back to these values of $N_{\text {eff }}$ at half filling in the discussion of the Drude weight. In order to examine the $\delta$-dependence of $N_{\text {eff }}$ near half filling, Fig. 4(b) depicts the $\delta$-dependence of $N_{\text {eff }} / \delta$ instead of raw $N_{\text {eff }}$. One can observe the linear behavior irrespective of the value of $U$ in the wide region of $\delta$. They extrapolate to finite 

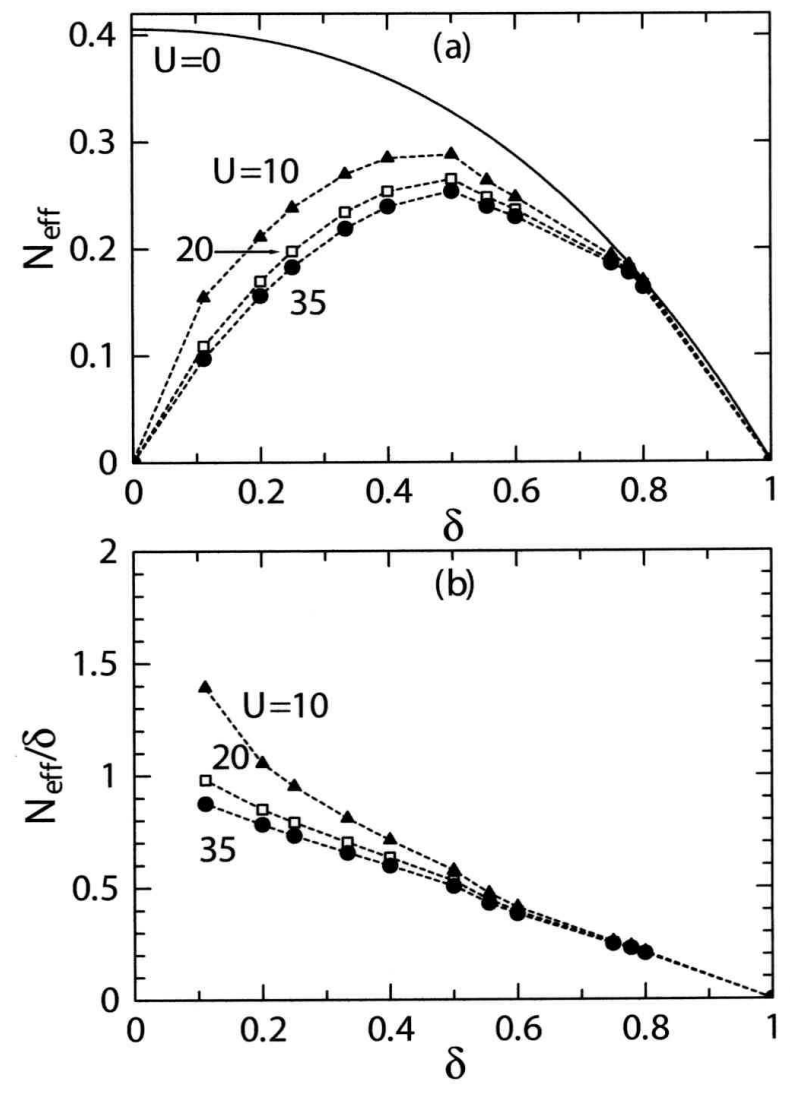

Fig. 4. $\delta$-dependence of $N_{\text {eff }}$ in (a) and $N_{\text {eff }} / \delta$ in (b). Closed triangles, open squares and closed circles denote data for $U=10$, 20 and 35 , respectively. In (a), total weight for $U=0$ is also depicted by the solid curve for comparison.

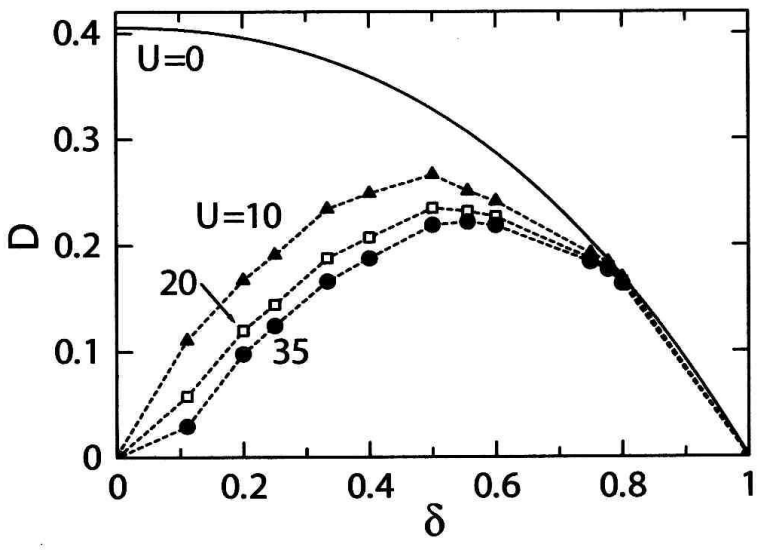

Fig. 5. $\delta$-dependence of Drude weight for $U=10,20$ and 35 . Notations for closed triangles, open squares and closed circles are the same as those in Fig. 4. For comparison, solid curve represents $D$ for $U=0$, which is the same as the total weight.

intercepts of the $N_{\text {eff }} / \delta$ axis, resulting in the following dependence

$$
N_{\text {eff }} \propto \delta,
$$

near half filling $\delta=0$. Note that the above dependence (6) is in agreement with all the previous studies. ${ }^{2-4}$

We depict $\delta$-dependence of the Drude weight in Fig. 5. Note that the Drude weight at $\delta=0$ vanishes for $U>0$

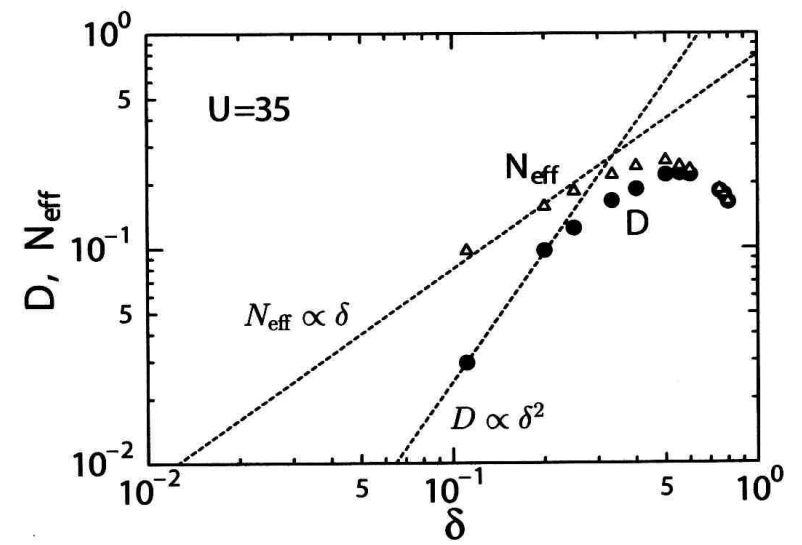

Fig. 6. Log-log plot of $D$ and $N_{\text {eff }}$ as function of $\delta$ for $U=35$. Circles and triangles denotes $D$ and $N_{\text {eff }}$, respectively.

in the thermodynamic limit. All the values for various $U$ are so small, less than 0.003 , that we cannot distinguish their symbols from the origin as shown in Fig. 4(a) and 5 , where our data of the half-filling case for $N_{\mathrm{s}}=20$ are plotted. For $N_{\mathrm{s}}=16$, we obtain $D \sim 0.009$ for $U=10$ at most; since its difference from the data of $N_{\mathrm{s}}=20$ is only within the symbol size, it is not shown in the figure. On the other hand, ref. 4 reported the Drude weight of about 0.02 , which is substantially larger than ours, for the cluster (c) with $N_{\mathrm{s}}=20$ under the periodic boundary condition. Our present result is closer to the correct value of zero in spite of the smaller size $N_{\mathrm{s}}$, which indicates superiority of our present calculations in reproducing well the insulating state at half filling.

In the metallic region for $\delta>0$, all the data obtained from studies on various system sizes are presented in Figs. 4(a) and 5 according to the discussion in section 3. Recall here that refs. 2 and 4 reported anomalous and discontinuous deviations of $D$ at some particular hole concentrations, resulting from a finite-size effect. ${ }^{11}$ On the other hand, the present data for all the values of $\delta$ in Fig. 5 show smooth variation on the whole, though they consist of mixture for various $N_{\mathrm{s}}$. It indicates that the finite-size effect is well eliminated in our estimates. As for the $\delta$-dependence of the Drude weight, the convex behavior is apparent in all the region of $\delta$ for $U=10$. For $U=20$ and 35, on the other hand, the concave behavior occurs near half filling. The change in this $\delta$-dependence with increasing $U$ will be discussed below.

In order to confirm the concave behavior of $D$, we plot the data of $D$ on the logarithmic scale in Fig. 6 together with $N_{\text {eff }}$ for $U=35$. Our data of $D$ near half filling indicate the dependence

$$
D \propto \delta^{2} .
$$

This dependence is in contrast to the linear dependence of $N_{\text {eff }}$ of eq. (6). For more detailed analysis of the dependence of $D$ in the region of small $\delta$, let us next examine the $\delta$-dependence of the ratio $D / N_{\text {eff }}$. The corresponding quantity was also studied in the two-dimensional $t-J$ model. ${ }^{12}$ The present results are shown in Fig. 7 in the form of $\left(D / N_{\text {eff }}\right) / \delta$. For $U=10$ and 20 , as $\delta$ is decreased toward half filling, the quantity $\left(D / N_{\text {eff }}\right) / \delta$ shows steep 


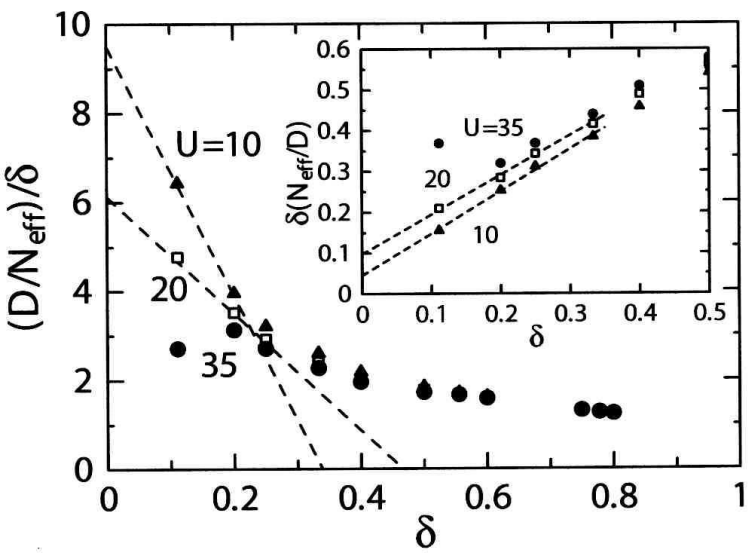

Fig. 7. $\delta$-dependence of $D /\left(N_{\mathrm{eff}} \delta\right)$ and its inverse. Notations for closed triangles, open squares and closed circles are the same as those in Fig. 4.

increase. For $U=35$, on the other hand, no such behavior is observed. The increasing behavior for $U=10$ and 20 is examined in the plot of the inverse of $\left(D / N_{\text {eff }}\right) / \delta$ shown in the inset of Fig. 7. Our data for $U=10$ and 20 reveal linear dependence in a wide region of $\delta$. For $U=35$, a similar linear dependence is observed in $0.2 \lesssim \delta \lesssim 0.5$ together with a deviation from it in the smaller- $\delta$ region. Each of the linear extrapolation of plots intersects at a non-zero intercept with the axis of ordinates in the limit of $\delta \rightarrow 0$. If one takes its weak $\delta$-dependence for $U=35$ into account, it is natural to consider that $\left[\left(D / N_{\text {eff }}\right) / \delta\right]^{-1}$ for various $U$ stays finite in the limit of $\delta \rightarrow 0$ as common behavior; there is no sign of vanishing $\left[\left(D / N_{\text {eff }}\right) / \delta\right]^{-1}$ from our data of finite size clusters. The above behavior, together with eq. (6), thus leads us to the scaling (7) valid not only for $U=35$ but also for $U=10$ and 20. This analysis is particularly important since it suggests the validity of the scaling (7) even for $U=10$ in spite of the fact that the $\delta$-dependence of $D$ for this $U$ in Fig. 5 does not show concave behavior which appears for $U=20$ and 35 .

Let us now discuss the present result from the viewpoint of the scaling theory developed by Imada, a general theory of the metal-insulator transition. ${ }^{13}$ This helps us to justify the dependence (7) in relation to other behavior of the two-dimensional Hubbard model. According to the scaling theory, the Drude weight shows the following critical behavior near the metal-insulator transition,

$$
D \propto \delta^{1+(z-2) / d},
$$

where $z$ denotes the dynamical exponent and $d$ is the spatial dimension. Since $d=2$ in the present problem, one obtains $z=4$ from (7). The scaling theory also predicts the critical behavior of the compressibility near the transition,

$$
\kappa \propto \delta^{1-z / d} .
$$

Substitution of $z=4$ and $d=2$ in the above dependence of $\kappa$ leads to $\kappa \propto \delta^{-1}$. The diverging behavior of $\kappa$ was reported by the quantum Monte Carlo (QMC) simulations $^{7,14}$ performed on the same model with larger sys-

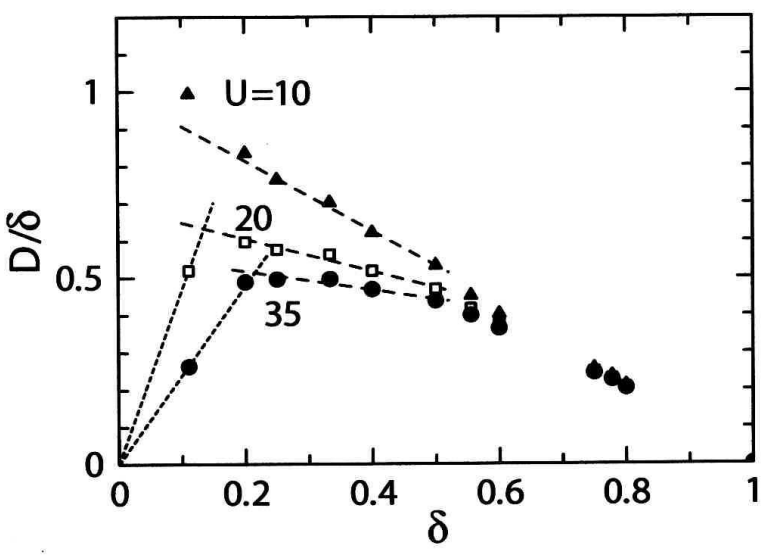

Fig. 8. $\delta$-dependence of $D / \delta$. Notations for closed triangles, open squares and closed circles are the same as those in Fig. 4. Broken lines are the fitting lines in $0.25 \leq \delta \leq 0.5$. Dotted lines are guides for eyes in the assumption of $D \propto \delta^{2}$.

tem sizes not yet treated by exact-diagonalization studies. The QMC calculation ${ }^{15}$ also demonstrated that the localization length suggests the divergence $\xi_{1} \propto\left|\mu-\mu_{\mathrm{c}}\right|^{-\nu}$ with $\nu=1 / 4$ as the chemical potential approaches the charge gap $\mu_{\mathrm{c}}$ from the insulating side. The scaling theory indicates $z \nu=1$, which also leads to the exponent $z=4$. Therefore, our present result is consistent with these QMC results in views of the scaling theory. Recent study of the metal-insulator transition ${ }^{16-18}$ clarified that the exponent $z=4$ appears at the marginal quantum critical point between the continuous metalinsulator transition with $T_{\mathrm{c}}=0$ and the discontinuous transition with nonzero $T_{\mathrm{c}}$. The appearance of the same marginal exponent $z=4$ was also found in the onedimensional Hubbard model with next-nearest-neighbor hopping. ${ }^{10,19}$ The unified understanding of the marginal point occurring in the one- and two-dimensional cases is the subject for future studies. Although it is difficult to determine the marginal quantum critical point precisely, the present results suggest that the critical region of the marginal point is wide extending to all the values of $U$ we studied. This allows us to capture successfully the distinct signature characteristic to the marginal point.

Next, let us consider the width of the critical region of $\delta$ in which the behavior (7) is observed. For this purpose, we depict the $\delta$-dependence of $D / \delta$ in Fig. 8 . In $0.25 \lesssim \delta \lesssim 0.5$, one can observe $U$-irrelevant linear behavior. In Fig. 8, we fit our numerical data in $0.25 \leq \delta \leq 0.5$ with broken lines. For $U=20$ and 35, our data near half filling digress from the broken lines. In particular, for $U=35$, two points shows clear deviation toward the origin in Fig. 8, while one point for $U=20$. Here, let us draw the dotted guide lines for these digressing data by assuming the present result (7). The dotted and the broken lines cross with each other for each $U$. One can regard the crossing point for each $U$ as a rough estimation of the boundary of the critical region. Then, the estimated boundaries are $\delta \sim 0.14$ and 0.22 for $U=20$ and 35, respectively. Though crude are these estimations, this is the first study to report the bound- 
ary. Calculations of systems with larger $N_{\mathrm{s}}$ with proper treatment of finite-size effects will make the precise estimations possible in future studies. For $U=10$, there are no data points showing digressing towards the origin. If the $\delta^{2}$ dependence of $D$, i.e. eq. (7), is true, then the above fact suggests that the critical region for $U=10$ is narrower than $\delta<1 / 9$. This is consistent with the rapid decrease of the estimated boundary as $U$ decreases from 35 to 20 . The doping concentration at the extrapolated boundary for $U=10$ may be very small.

Let us discuss the frequency dependence of $\sigma^{\mathrm{reg}}(\omega)$ obtained by eq. (2) for the insulating and metallic cases where the system is in the critical region; we present our results in Fig. 9. Figures 9(a) and 9(b) depict the results for insulating cases. The structures of $\sigma^{\mathrm{reg}}(\omega)$ between $N_{\mathrm{s}}=16$ and $N_{\mathrm{s}}=20$ are similar for each $U$. This fact suggests that finite-size effects are rather irrelevant for the structure of $\sigma^{\text {reg }}(\omega)$. For each $U$, broad responses corresponding to the transitions to the upper-Hubbard band appear around $\omega=U$. For $U=10$, a sharp peak emerges at the lower edge of the band. According to ref. 4 , this sharp peak is ascribed to the spin-polaron formation in the photoexcited state, and the weight of the peak becomes smaller for larger $N_{\mathrm{s}}$. On the other hand, our sharp peak for $U=10$ does not show any significant $N_{\mathrm{s}}$ dependence, which implies that it will survive in the thermodynamic limit, although the peaks get rapidly weaker for larger $U=20$ and 35 . The broad responses are unaffected by the change in $U$ between $U=20$ and 35 except that the center of responses moves with $U$. Figures $9(\mathrm{c})$, $9(\mathrm{~d})$ and $9(\mathrm{e})$ depict the metallic cases; one can observe not only the high-frequency spectrum due to the transitions to the upper-Hubbard band but also a pronounced structure in the low-energy region below the Mott gap. First, let us see the transitions to the upper-Hubbard band. By switching $\delta$ on, these transitions gradually lose their weights by transferring them to the lower-energy structure, as already been reported in refs. 2, 3 and 4 . Next, let us see the structure in the low-frequency region. We find strong responses growing rapidly towards $\omega=0$ with decreasing $\omega$. It is noticeable that the shape seems to be a common property irrespective of $U$ and $\delta$ within the present parameter ranges. The same shapes are produced in the present results of both $N_{\mathrm{s}}=18$ and 20 . The $\omega$-dependence of the shape reminds us of the $1 / \omega$ tail and the mid-IR peak observed in the experiments of high- $T_{\mathrm{c}}$ compound. ${ }^{20}$

Finally, let us make comparisons with experimental results of optical conductivities. Recall here that the coherent and incoherent parts are not definitely separated in the experimentally observed optical conductivity. Thus, it is not so easy to make direct comparison between experimental and numerical $\sigma^{\text {reg }}(\omega)$. Theoretically, on the other hand, one has to introduce the broadening of delta functions with some widths in dealing with finite systems as the present ones. Keeping these circumstances in mind, we consider the frequency dependence of $\sigma(\omega)$ including the Drude part; Figure 10 depicts results of $U=10$, typical value of the Coulomb interaction for most of the high- $T_{\mathrm{c}}$ materials. For $\delta=0$, the present spectrum reveals a clear increase as $\omega \rightarrow 0$, even when
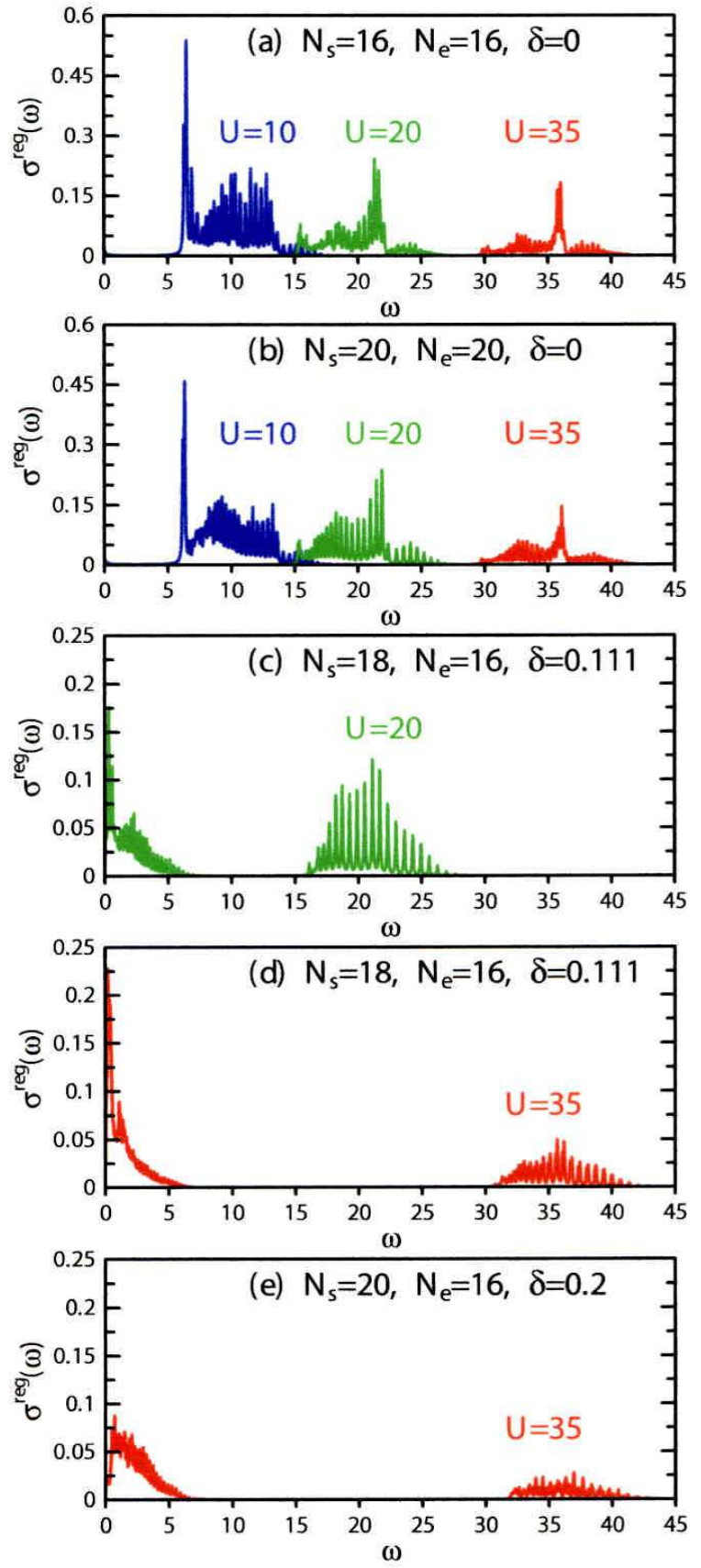

Fig. 9. Frequency dependence of regular part of the optical conductivity in (a) for $N_{\mathrm{s}}=16$ and $N_{\mathrm{e}}=16$, namely $\delta=0$, (b) for $N_{\mathrm{s}}=20$ and $N_{\mathrm{e}}=20$, namely $\delta=0$, (c) for $N_{\mathrm{s}}=18$ and $N_{\mathrm{e}}=16$, namely $\delta=1 / 9$, (d) for $N_{\mathrm{s}}=18$ and $N_{\mathrm{e}}=16$, namely $\delta=1 / 9$ and (e) for $N_{\mathrm{s}}=20$ and $N_{\mathrm{e}}=16$, namely $\delta=0.2$. Blue, green and red lines denote the cases for $U=10, U=20$ and $U=35$, respectively. Delta functions are broadened with width of $\eta=0.05$.

the system is in the insulating state. This comes from the tiny $D$ due to the finite-size effect. For $\delta>0$, on the other hand, the Drude weight is considerably large in comparison with this tiny $D$ at half filling; there occurs a huge peak at $\omega=0$ as the coherent component. Its tail in Fig. 10 appears due to the broadening of width $\eta=0.2$. Note here that even the tail is sizable in comparison with the intrinsic responses of $\sigma^{\mathrm{reg}}(\omega)$ in the innergap region. This means that in order to understand op- 


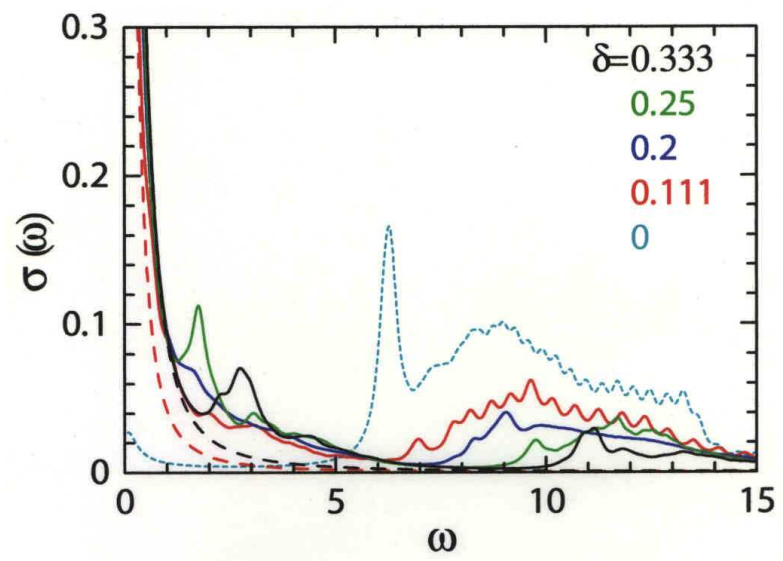

Fig. 10. Frequency dependence of optical conductivities for $U=$ 10. The insulating case of $N_{\mathrm{s}}=20$ and $N_{\mathrm{e}}=20$ is denoted by the sky-blue dotted line. Red, deep-blue, green and black solid lines correspond to the metallic cases of $N_{\mathrm{s}}=18$ and $N_{\mathrm{e}}=16$, $N_{\mathrm{s}}=20$ and $N_{\mathrm{e}}=16, N_{\mathrm{s}}=16$ and $N_{\mathrm{e}}=12$ and $N_{\mathrm{s}}=18$ and $N_{\mathrm{e}}=12$, respectively. Delta functions are broadened with width of $\eta=0.2$. For comparison, the coherent part in $\sigma(\omega)$ is also presented for the two cases of $N_{\mathrm{s}}=18$ by the broken lines of the same colors.

tical responses of the system, it is important to capture both the behavior of the coherent component and that of the incoherent one included in the total $\sigma(\omega)$ simultaneously. In Fig. 10, qualitatively the same change by doping in the transitions to the upper-Hubbard band is seen as that mentioned above for $U=20$ and 35. The structure in this $\omega$ region should be associated with the charge transfer from the copper $d$ to the oxygen $p$ states in the case of the cuprates, hence it is, in narrow sense, different from the present case of the Mott-Hubbard type. Aside from the character of the gap, i.e., either it is the Mott-Hubbard gap or the charge transfer gap, the evolution of doping observed in the experiments are correctly captured in our numerical results, the spectral weight transfer from the higher to lower energies as well as the collapse of the sharp peak at the low-energy edge.

The evolution of the low-energy structure with doping contains both the growth of the Drude weight and the development of the incoherent mid-IR structure. The total sum of these two contributions is the effective carrier density $N_{\text {eff }}$, whose weight is proportional to the doping concentration. In the doping process of the evolution from the insulator to low doping, the mid-IR incoherent part below the Mott gap grows quickly in our calculated results and is already prominent even in underdoped systems (see the result at $\delta \sim 0.111$ ), with a rather insensitive dependence on the further doping to the overdoped systems ( see $\delta=0.333$ ). On the contrary to the mid-IR part, the Drude part grows slowly at low doping, while it starts growing quickly from moderate to over doping, which is the origin of the scaling given by $D \propto \delta^{2}$ rather than $D \propto \delta$. The total sum resulting in $N_{\text {eff }}$ evolves smoothly and linearly with $\delta$ because of the compensation of these two contributions. The fact that the Drude part remains only a small fraction of $N_{\text {eff }}$ whereas the mid-IR structure rapidly grows from zero to low dopings is indeed common to all the high- $T_{\mathrm{c}}$ cuprates. It is even more universal in the transition metal oxides in general. Our calculated result for the further doping process from moderate to over doping is also consistent with the universal trend of the experimental results in transition metal oxides including the high- $T_{\mathrm{c}}$ cuprates, where the mid-IR structure is absorbed to the Drude weight. The $\delta^{2}$ scaling of the Drude weight is tightly related to this profound and universal trend in the doping process of the whole spectral weight in the optical conductivity.

\section{Conclusion and Discussion}

We have investigated the Drude weight of the Hubbard model on the square lattice by means of large-scale parallelized exact diagonalizations. The hole-doping dependence of the Drude weight near the transition point between the metal and the Mott insulator has been a controversial problem. We have very carefully examined finite-size effects and we have successfully captured the intrinsic behavior of the Drude weight. Our results suggest that the Drude weight is scaled by $D \propto \delta^{2}$, which is consistent with results from various kinds of analysis. In particular, it is important that this scaling agrees with the quantum Monte Carlo results through the scaling theory of the metal-insulator transition. The behavior $D \propto \delta^{2}$ is a characteristic feature near the marginal quantum critical point of the transition between the Mott insulator and the doped metal in two-dimensional systems. The restructuring of the electronic structure upon doping into the Mott insulator first causes the spectral weight transfer from the weight above the gap mainly to a mid-IR incoherent component below the gap. The spectral weight is further progressively transferred from the mid-IR component to the Drude weight. This largescale restructuring seen in the frequency and doping dependences of $\sigma(\omega)$ in our result is consistent with the experimental results on transition metal oxides including high- $T_{\mathrm{c}}$ cuprates. The $\delta^{2}$ scaling of the Drude weight comes from this underlying physics involving the high energy scale.

At $U=0$, the Hubbard model we studied has a perfect nesting just at half filling and the Fermi level coincides with the van Hove singularity. The effect of deviation from this perfect nesting by introducing the hopping to further sites (such as the next-nearest-neighbor hopping $\left.t^{\prime}\right)$ is left for separate study. The QMC study ${ }^{14}$ indicates that the scaling behavior of the compressibility does not sensitively depend on the presence or absence of $t^{\prime}$, which implies that the present scaling of the Drude weight also holds. Next, let us recall the marginal point with $z=4$ in the one-dimensional Hubbard model with the nextnearest-neighbor hopping ${ }^{10,19}$ mentioned above. In this case, a singularity in the density of states is absent at half filling at $U=0$ because the next-nearest-neighbor hopping is small. This fact also suggests that the metalinsulator transition with $z=4$ occurs irrespective of such a singularity in the density of states.

The finite-size effects in such a quantity as the Drude weight are considerably large. Thus, the careful examination of the finite-size effects is necessary in possible ways as much as we can. If one calculates the quantity 
without considering the effects, obtained results could severely suffer from superficial finite-size effects. By such results, unfortunately, the incorrect conclusion would be derived. In this meaning, the present problem of the Drude weight is an instructive example. As a way to reduce the finite-size effect, the present work employs appropriately chosen clusters under the appropriate boundary conditions. Thereby, we have captured the critical behavior of $D \propto \delta^{2}$ near the half-filling insulator. Averaging over twisted boundary conditions ${ }^{21}$ may be another possibility for the reduction. Quite recently such an investigation has been done in the $N_{\mathrm{s}}=20$ cluster of the $t$ - $J$ model plus three-site terms. ${ }^{22}$ Although this work gives a result of the $\delta$-dependence of $D$, it is so subtle that one cannot conclude either of $D \propto \delta^{2}$ or $D \propto \delta$. In the not-too-distant future, computers will be even more powerful; numerical diagonalizations of the Hubbard model with larger system sizes would be possible. Such calculations could clarify the validity of the present procedure for reducing the finite-size effects in $D$ and provide us with a more definite result for the critical behavior of $D$. We hope that the knowledge in the present study will serve for such future studies.

\section{Acknowledgements}

This work was supported by a Grant-in-Aid for Young Scientists (B) from the Ministry of Education, Culture, Sports, Science and Technology of Japan (15740221). A part of the computations was performed using facilities of the Information Initiative Center, Hokkaido University and the Supercomputer Center, Institute for Solid State Physics (ISSP), University of Tokyo. The authors thank Prof. Hajime Takayama and the staffs of the Supercomputer Center, ISSP for their support to perform our large-scale parallelized calculations in a special job class of the supercomputer of ISSP.

1) M. Imada, A. Fujimori and Y. Tokura: Rev. Mod. Phys. 70 (1998) 1039 and references therein.
2) E. Dagotto, A. Moreo, F. Ortolani, D. Poilblanc and J. Riera: Phys. Rev. B 45 (1992) 10741.

3) H. Nakano and M. Imada: J. Phys. Soc. Jpn. 68 (1999) 1458.

4) T. Tohyama, Y. Inoue, K. Tsutsui and S. Maekawa: Phys. Rev. B 72 (2005) 045113.

5) The largest dimension of the subspace of the Hubbard model with a given $N_{\mathrm{s}}$ is realized at half filling with the same number of up-spin electrons and down-spin ones. For examples, the dimensions are 165636900 for $N_{\mathrm{s}}=16,2363904400$ for $N_{\mathrm{s}}=18$ and 34134779536 for $N_{\mathrm{s}}=20$. It is possible to reduce these dimensions by using the symmetries of the Hamiltonian; however, the program for the reduced case is not necessarily applicable to any Hamiltonians having arbitrary shapes of clusters in contrast to our program. The versatile feature of our code makes it possible to examine the various cases very easily. Although the dimension is very huge, our code costs 29.7 hours for the 260-time iterations to obtain the ground-state energy of the 20-site half-filling Hamiltonian for $U=35$, when we use 16 nodes in the supercomputer SR11000 in Hokkaido University.

6) E. Gagliano and C. Balseiro: Phys. Rev. Lett. 59 (1987) 2999.

7) N. Furukawa and M. Imada: J. Phys. Soc. Jpn. 60 (1991) 3604; ibid.61 (1992) 3331.

8) H. Nakano and Y. Takahashi: J. Phys. Soc. Jpn. 72 (2003) 1191.

9) H. Nakano and Y. Takahashi: J. Mag. Mag. Mat. 272-276 (2004) 487.

10) H. Nakano and Y. Takahashi: J. Phys. Soc. Jpn. 73 (2004) 983.

11) The negative Drude weights with large absolute values were reported at $\delta=0$ and $\delta=1 / 8$ for $N_{\mathrm{s}}=16$ in ref. 2 . In ref. 4 , the discontinuous dependence was reported at $\delta=1 / 3$ for $N_{\mathrm{s}}=18$. These works use only the periodic boundary condition. Note that such behavior occurs only at the hole concentration when the electronic shell structure is open.

12) H. Tsunetsugu and M. Imada: J. Phys. Soc. Jpn. 67 (1998) 1864.

13) M. Imada: J. Phys. Soc. Jpn. 64 (1995) 2954.

14) N. Furukawa and M. Imada: J. Phys. Soc. Jpn. 62 (1993) 2557.

15) F. Assaad and M. Imada: Phys. Rev. Lett. 76 (1996) 3176.

16) M. Imada: J. Phys. Soc. Jpn. 74 (2005) 859.

17) M. Imada: Phys. Rev. B 72 (2005) 075113.

18) T. Misawa, Y. Yamaji and M. Imada: J. Phys. Soc. Jpn. 75 (2006) 083705.

19) H. Nakano, Y. Takahashi and M. Imada: Physica B 359-361C (2005) 657.

20) S. Uchida, T. Ido, H. Takagi, T. Arima, Y. Tokura and S. Tajima: Phys. Rev. B 43 (1991) 7942.

21) D. Poilblanc: Phys. Rev. B 44 (1990) 9562.

22) T. Tohyama: J. Phys. Chem. Solids 67 (2006) 2210. 